\title{
Mucosal immunity: aliment and ailments
}

$\mathrm{ERaz}^{1}$

The development of the primitive gut in the multicellular aquatic eukaryote was driven by the insufficient absorption of nutrients from the oceanic soup. The anatomy of this evolving specialized system invited its colonization by environmental prokaryotes and resulted in the establishment of the intestinal microflora. Innate immunity had previously evolved in gutless organisms such as plants and was fully functional in the gut of invertebrates. On the other hand, adaptive immunity evolved in vertebrates, most probably because of some selective pressure such as the adaptation to a predatory lifestyle. Interestingly, this form of immunity was localized first in their primitive gut. Although the newly generated eukaryote-prokaryote relationship in the gut evolved under mutualistic principles, its symbiotic nature is easily interrupted by extrinsic factors such the composition of the consumed food. Thus, it is argued below that a state of disequilibrium with the microflora (dysbiosis) results in potentially serious immune-related disadvantages to the host.

\section{THE PRIMITIVE GUT ANDTHE ORIGIN} OF THE MICROFLORA

The growth and evolution of ancient, aquatic, blastula-like metazoans reached a stationary phase when endophagocytosis of environmental nutrients from the primordial soup was insufficient to sustain life. ${ }^{1,2}$ Subsequently, a decisive evolutionary step took place that led to the formation of a true epithelium and gastrulation in eumetazoans, providing a more efficient nutritive system. ${ }^{2}$ This system, which evolved to the primitive gut in higher species, could capture and extract high volumes of nutrients, including various prokaryotes from the primordial aquatic soup. This change in the feeding process led to the colonization of the gut tube by certain environmental bacterial species or the primary colonizers. Owing to their microbial activities in the gut, the primary colonizers generated conditions suitable for colonization by other species, known as the secondary colonizers (i.e., autogenic succession). ${ }^{3}$ Additional changes were enforced in the composition of this early microbial community by external, non-microbial factors (i.e., allogenic succession). The increases in complexity of the various autogenic and allogenic interactions contributed to the generation of a stable microflora and a unique eukaryote-prokaryote ecosystem. ${ }^{4}$ The subsequent co-evolution of the host with its microflora was driven by mutualistic principles under which both partners benefit from their interaction. In this setting, the host provides the "perfect incubator", whereas the microflora provides, among other things, its fermenting or enzymatic machinery that enhances the efficiency of nutrient extraction, especially complex carbohydrates, from the lumen of the primitive gut tube.

THE PRIMITIVE GUT: ITS RELATIONSHIP WITH INNATE AND ADAPTIVE IMMUNITY Certain species of microorganisms from the microflora are capable of infecting their host, as are other opportunistic bacteria. In lower species, this bacterial expansion beyond the gastrointestinal tube is met with a defense mechanism known as innate immunity. Innate immunity is the main anti-microbial defense mechanism in plants and invertebrates, and is triggered by various microbial ligands that are recognized in the host by pattern recognition receptors (PRRs). ${ }^{5}$ The signature effector molecules of this defense system in invertebrates are antimicrobial molecules such as lysozyme, complement factors, lectins, and antimicrobial peptides. In contrast to innate immunity, adaptive immunity evolved only in vertebrates and functions as an important part of their defense system. The effector molecules of adaptive immunity, immunoglobulins and TCRs, are produced by lymphocytes that undergo clonal selection, expansion, and retain antigenic memory, all triggered by microbial antigens. ${ }^{6}$ Interestingly, this type of adaptive immune system has not been found in jawless vertebrates that developed a different adaptive immune system. ${ }^{7}$ Therefore, the evolution of the jaw structure in primitive fish might hint at, or

${ }_{1}^{1}$ Department of Medicine 0663, University of California, San Diego, La Jolla, California, USA. Correspondence: E Raz (eraz@ucsd.edu) 
even explain, the emergence of adaptive immunity as a system complementary to innate immunity. It has been further postulated that adaptive immunity evolved in primitive jawed fish owing to a newly acquired predatory feeding style, which is used by larger organisms for a more efficient nutrient extraction. ${ }^{8}$ The role of the newly evolved adaptive immunity was most likely to protect the host from repeated gastrointestinal injuries and the consequent infections originating from the spreading of intestinal bacteria to the adjacent tissues, presumably through the activation of its memory cells. Morphological studies support the assumption that the primitive adaptive immune system was generated in the hematopoietic tissues, primarily in the intestinal tract. ${ }^{6,8}$ In mice and other mammals, the gut-associated lymphoid tissue (GALT) is most likely represented by lymphoid aggregates in the lamina propria that are also known as cryptopatches. These cryptopatches are thymus-like, mini-lymphoid organs that drive IL-7-dependent lymphopoiesis. ${ }^{9}$

Taken together, these observations confirm the impact of the evolutionary pressure imposed by a predatory lifestyle on the development of adaptive immunity primarily as a defense system of the gastrointestinal tract (i.e., mucosal immunity). It also suggests that the emerging gastrointestinal milieu is the alma mater of adaptive immune cells in higher species. It is interesting to note that establishment of the gastrointestinal tract and predatory skills combined with the development of the respiratory system were essential and mandatory for primitive amphibians, and later for other species, to thrive after changing from an aquatic to terrestrial habitat.

\section{THE GASTROINTESTINAL TRACT:TISSUE} ADAPTATION OF INNATE IMMUNITY

The human gastrointestinal tract harbors more than 500 distinct microbial species totaling an estimated $10^{14}$ microbes, which together contain approximately 100 -fold more genes than their human host. ${ }^{10}$ This vast symbiotic microbial consortium (also known as the forgotten organ) is initiated in each person immediately during or after birth and, following autogenic and allogenic successions, reaches its stable composition during early infancy. This intriguing and universal colonization indicates that there is a process utilized by the host to select various microbial species that results in a successful and beneficial colonization of the gastrointestinal tract.

As we discussed above, innate immunity did not evolve to protect the host solely from intestinal microflora. Indeed, the genetic deletion of MyD88, the main adaptor protein of the Toll-like receptors (TLRs), does not increase the occurrence of spontaneous intestinal inflammation. Other contributions of the microfloraactivated innate immunity to the homeostasis and functions of the gastrointestinal tract were addressed recently in a series of studies. Collectively, these studies revealed that activation of innate immunity (1) affects the composition of the intestinal microflora, ${ }^{11}$ (2) enhances the integrity and barrier functions of intestinal epithelial cells (IECs) via the induction of tight junction-related proteins, ${ }^{12}$ (3) increases the proliferative response and decreases the pace of apoptosis of IECs, as was inferred from studies in wildtype mice treated with TLR agonists ${ }^{13}$ or various TLR knockout mice, ${ }^{14,15}$ and (4) accelerates the healing process of the injured epithelium. ${ }^{16}$ Finally, activation of innate immunity via Nod or TLR results in the secretion of anti-microbial peptides, ${ }^{17}$ and triggering of Nod1 supports GALT via the generation of isolated lymphoid follicles (ILFs) in the intestinal mucosa. ${ }^{18}$

Taken together, these observations highly suggest that activation of innate immunity at the host-microbial interface of the gastrointestinal tract has adapted from inducing inflammation (tissue damage) to healing and restitution (tissue building). ${ }^{19}$ Hence, the overall protective effect of innate immunity on the host is through the maintenance of IEC integrity, which is mandatory for the proper metabolic functions of the gastrointestinal tract. Mechanistically, this tissue adaptation in the function of innate immunity can be largely explained by the activation of PRR-bearing IECs by microbial ligands derived from the microflora ${ }^{20}$ and by the unique ability of these cells to undergo polarization, which modifies inflammatory TLR activation. ${ }^{21}$

\section{FROM SYMBIOSIS TO DYSBIOSIS}

The cultivation and domestication of grains such as wheat, rye, rice, and maize, and early farming communities, began across the globe around 10,000 years ago, and likely imposed a radical change in the composition of the microflora by giving growth advantages (i.e., a prebiotic effect) to bacterial species that are capable of digesting complex carbohydrates (e.g., Firmicutes spp). The domestication of herd animals such as cattle, sheep, and goats to livestock was initiated in Mesopotamia 9,000 years ago. ${ }^{22}$ Animal domestication resulted in the consumption of higher volumes of dairy products throughout life by certain human communities (especially in Mesopotamia and Europe). ${ }^{22}$ This revolution in diet altered both their metabolism and their microflora (e.g., lactase and Lactobacillus spp, respectively). The change in lifestyle from hunter-gatherer to farming, agriculture-based and, later, to urban living most likely introduced microbial alterations due to novel feeding habits and new inhabitation styles. The international food trade and novel culinary customs initiated a few hundred years ago helped the dissemination of new food products (e.g., sugar, corn, spices) and had modified feeding habits on a global scale. Furthermore, recent cultural and technological advances have also introduced novel hygiene practices, which indiscriminately affect the composition of the microflora.

The "Old Friends" theory suggests that owing to these cultural changes and technological progress, the composition of the microflora has adapted. Harmless environmental saprophytes (the "old friends") that are present in overwhelming amounts in soil, food, water, and domesticated animals were routinely consumed with food and water. These pseudo-commensals were therefore part of the human microflora for thousands of years. ${ }^{23}$ This long-standing relationship, which had to be tolerated by the host, was then replaced by potentially "unfriendly" and less adapted microbial species. Similar arguments can also be 
made for certain helminthes. This change in microflora composition has been intensely accelerated in industrialized countries over the last few decades, most likely because of the decrease in human milk and the increase in formula feedings of infants, and because of the consumption of overprocessed and overly purified food, clean water, and the use of antibiotics (i.e., hygiene practices). ${ }^{24}$ In fact, these modern feeding habits bypass to some extent the need for microbial help in nutrient extraction. Furthermore, they provide growth advantages to certain microbial species and growth disadvantages to others, thus generating an unstable intestinal ecosystem or dysbiosis. If proven by modern molecular and DNA-sequencing techniques, this ongoing trend can generate a drift, or a shift, in the host-microbial relationship, thereby supporting the transition to real commensalisms, in which only the microflora benefits, while selecting against mutualism or symbiosis, in which both the host and the microflora benefit. Of most concern are changes in the composition of the microflora that can be very influential in early infancy when both intestinal colonization and development of the adaptive immune system and its memory responses occur.

\section{DYSBIOSIS AND ITS POTENTIAL CONSEQUENCES ON HUMAN HEALTH}

As argued in the previous sections, the proper maintenance of the intestinal microbial consortium results in metabolic and immunological benefits and, therefore, can be of great importance for human health. ${ }^{25}$ Thus, it is possible that a dysbiotic microflora (e.g., lacking the "old and faithful" bacteria and helminths) leads to a dysregulation of adaptive immunity. ${ }^{23}$ A dysbiotic relationship may inhibit the proper balance between regulatory and effector $\mathrm{T}$ cells and encourages the imprinting and differentiation of inflammatory effector immune cells (e.g., Th1, Th2, or Th17 subsets), as was described in two recent studies. The first study documented that a polysaccharide A derived from Bacteroides fragilis is capable of regulating infectious colitis by the inhibition of IL-17-producing $\mathrm{CD} 4+\mathrm{T}$ cells and the induction of
IL-10-producing CD4 + T cells. ${ }^{26}$ The second study showed that Th17 cell differentiation in the small intestine of normal mice requires specific commensals (cytophaga-flavobacter-bacteroidetes) and can be inhibited by selective antibiotic treatment. Furthermore, the reduction of Th17 cell-inducing bacteria in this study was accompanied by an increase in regulatory T cells. ${ }^{27}$ This microbial-driven immune imbalance may contribute to the observed increase in prevalence of various organ-specific, immune-mediated inflammatory diseases such as asthma, multiple sclerosis, ${ }^{28}$ and, more direct for our discussion, inflammatory bowel disease. ${ }^{29}$ This theory, which stems from the "Hygiene Hypothesis", ${ }^{30,31}$ is supported by a few recent clinical and experimental data, through which the role of microflora in the modulation of immunemediated inflammatory disorders was validated. Indeed, the administration of different probiotic bacterial preparations inhibits intestinal inflammation in patients who suffer from a certain clinical subset of ulcerative colitis. ${ }^{32}$ Conversely, a change in composition of the microbiota has been shown to provoke either type-1 diabetes in genetically prone animals ${ }^{11}$ or experimental colitis in other murine models. ${ }^{33}$ Mechanistically, a dysbiotic relationship may allow microbial translocation from the gut lumen, leading to a sustained immune activation at local mucosal and submucosal sites. This overstimulated immune response can lead to the subsequent induction of inflammatory and autoimmune conditions. Alternatively, a dysbiotic relationship may shape dendritic cells to prime an over-reacting and inflammatory adaptive T-cell response.

Taken together, these observations, although insufficient, suggest the imprinting of a regulatory tone for the symbiotic microflora on adaptive immunity, especially in early life. Understanding the molecular and cellular basis of these effects, from both the microbial and host perspectives, would most likely provide us with a variety of tools (e.g., functional food, prebiotic, and postbiotic preparations) that can prevent or modulate chronic inflammatory and incapacitating human disorders.

\section{ACKNOWLEDGMENTS}

This work was supported by $\mathrm{NIH}$ grants Al068685, Al083328, DK35108, and a grant from Crohn's \& Colitis Foundation of America.

\section{DISCLOSURE}

The author declared no conflict of interest.

() 2010 Society for Mucosal Immunology

\section{REFERENCES}

1. Neish, A.S. Microbes in gastrointestinal health and disease. Gastroenterology 136, 65-80 (2009).

2. Nielsen, C. Six major steps in animal evolution: are we derived sponge larvae? Evol. Dev. 10, 241-257 (2008).

3. Adlerberth, I. Factors influencing the establishment of the intestinal microbiota in infancy. Nestle Nutr. Workshop Ser. Pediatr. Program 62, 13-29; discussion 29-33 (2008).

4. Salonen, A., Palva, A. \& de Vos, W.M. Microbial functionality in the human intestinal tract. Front. Biosci. 14, 3074-3084 (2009).

5. Nurnberger, T., Brunner, F., Kemmerling, B. \& Piater, L. Innate immunity in plants and animals: striking similarities and obvious differences. Immunol. Rev. 198, 249-266 (2004).

6. Pancer, Z. \& Cooper, M.D. The evolution of adaptive immunity. Annu. Rev. Immunol. 24, 497-518 (2006).

7. Guo, P. et al. Dual nature of the adaptive immune system in lampreys. Nature 459 , 796-801 (2009).

8. Matsunaga, T. \& Rahman, A. What brought the adaptive immune system to vertebrates?--The jaw hypothesis and the seahorse. Immunol. Rev. 166, 177-186 (1998).

9. Rocha, B. The extrathymic T-cell differentiation in the murine gut. Immunol. Rev. 215, 166-177 (2007).

10. Backhed, F., Ley, R.E., Sonnenburg, J.L., Peterson, D.A. \& Gordon, J.I. Host-bacterial mutualism in the human intestine. Science 307, 1915-1920 (2005).

11. Wen, L. et al. Innate immunity and intestinal microbiota in the development of Type 1 diabetes. Nature 455, 1109-1113 (2008).

12. Cario, E., Gerken, G. \& Podolsky, D.K. Toll-like receptor 2 controls mucosal inflammation by regulating epithelial barrier function. Gastroenterology 132, 1359-1374 (2007).

13. Rachmilewitz, D. et al. Immunostimulatory DNA ameliorates experimental and spontaneous murine colitis. Gastroenterology 122, 1428-1441 (2002).

14. Rakoff-Nahoum, S., Paglino, J., EslamiVarzaneh, F., Edberg, S. \& Medzhitov, R. Recognition of commensal microflora by toll-like receptors is required for intestinal homeostasis. Cell 118, 229-241 (2004).

15. Fukata, M. et al. Toll-like receptor-4 is required for intestinal response to epithelial injury and limiting bacterial translocation in a murine model of acute colitis. Am. J. Physiol. Gastrointest. Liver Physiol. 288, G1055-G1065 (2005).

16. Fukata, M. \& Abreu, M.T. TLR4 signalling in the intestine in health and disease. Biochem. Soc. Trans. 35, 1473-1478 (2007).

17. Uehara, A., Fujimoto, Y., Fukase, K. \& Takada, $H$. Various human epithelial cells express functional Toll-like receptors, NOD1 and NOD2 to produce anti-microbial peptides, but not 
proinflammatory cytokines. Mol. Immunol. 44, 3100-3111 (2007).

18. Bouskra, D. et al. Lymphoid tissue genesis induced by commensals through NOD1 regulates intestinal homeostasis. Nature 456, 507-510 (2008).

19. Raz, E. Organ-specific regulation of innate immunity. Nat. Immunol. 8, 3-4 (2007).

20. Lee, J. et al. Maintenance of colonic homeostasis by distinctive apical TLR9 signalling in intestinal epithelial cells. Nat. Cell Biol. 8, 1327-1336 (2006).

21. Lee, J., Gonzales-Navajas, J.M. \& Raz, E. The "polarizing-tolerizing" mechanism of intestinal epithelium: its relevance to colonic homeostasis. Semin. Immunopathol. 30, 3-9 (2008).

22. Evershed, R.P. et al. Earliest date for milk use in the Near East and southeastern Europe linked to cattle herding. Nature 455, 528-531 (2008).

23. Rook, G.A. Review series on helminths, immune modulation and the hygiene hypothesis: the broader implications of the hygiene hypothesis. Immunology 126, 3-11 (2009).

24. Schaub, B., Lauener, R. \& von Mutius, E. The many faces of the hygiene hypothesis. J. Allergy Clin. Immunol. 117, 969-977; quiz 978 (2006).

25. Dethlefsen, L., McFall-Ngai, M. \& Relman, D.A. An ecological and evolutionary perspective on human-microbe mutualism and disease. Nature 449, 811-818 (2007).

26. Mazmanian, S.K., Round, J.L. \& Kasper, D.L. A microbial symbiosis factor prevents intestinal inflammatory disease. Nature 453, 620-625 (2008).

27. Ivanov, II et al. Specific microbiota direct the differentiation of IL-17-producing T-helper cells in the mucosa of the small intestine. Cell Host Microbe 4, 337-349 (2008).

28. Noverr, M.C. \& Huffnagle, G.B. Does the microbiota regulate immune responses outside the gut? Trends Microbiol. 12, 562-568 (2004).
29. Koloski, N.A., Bret, L. \& Radford-Smith, G. Hygiene hypothesis in inflammatory bowel disease: a critical review of the literature. World J. Gastroenterol. 14, 165-173 (2008).

30. von Mutius, E. Allergies, infections and the hygiene hypothesis - the epidemiological evidence. Immunobiology 212, 433-439 (2007).

31. Rook, G.A. The hygiene hypothesis and the increasing prevalence of chronic inflammatory disorders. Trans. R. Soc. Trop. Med. Hyg. 101, 1072-1074 (2007).

32. Schultz, M. \& Lindstrom, A.L. Rationale for probiotic treatment strategies in inflammatory bowel disease. Expert Rev. Gastroenterol. Hepatol. 2, 337-355 (2008).

33. Kim, S.C. et al. Variable phenotypes of enterocolitis in interleukin 10-deficient mice monoassociated with two different commensal bacteria. Gastroenterology 128, 891-906 (2005). 